\title{
LOS ESTUDIANTES UNIVERSITARIOS ANTE LAS ACTIVIDADES EXTRACURRICULARES ${ }^{1}$
}

\section{UNIVERSITY STUDENTS AND EXTRACURRICULAR ACTIVITIES}

\author{
José Rodrigo Pozón López \\ Universidad Anáhuac México Sur \\ jrpozon@yahoo.es, jose.pozon@anahuac.mx
}

\begin{abstract}
Resumen: Este estudio aborda el fenómeno del involucramiento estudiantil en actividades extracurriculares, utilizándose para ello una estrategia esencialmente de corte cualitativo, con la participación de estudiantes, profesores y administrativos en grupos focales, entrevistas y un cuestionario, haciendo posible: conocer valoraciones sobre el fenómeno; averiguar qué factores lo influyen; detectar sus consecuencias; e identificar qué prioridad se otorga a cada actividad. El estudio se realizó en una universidad privada católica de elite de la Ciudad de México.
\end{abstract}

Palabras clave: Involucramiento estudiantil, actividades extracurriculares, formación integral, experiencia universitaria.
Abstract: With the purpose of exploring the phenomenon of student involvement in extracurricular activities, we used a qualitative approach methodology in which students, teachers and administrative staff participated in focus groups, interviews and a questionnaire. This made it possible for us to learn about evaluating the phenomenon, know which factors affect it, detect its consequences, and identify what priority each activity has for the students. This study was conducted at an elite, Catholic, private university in Mexico City.

Key words: Student engagement, extracurricular activities, integral formation, college experience.

1 Este trabajo ha sido realizado bajo el asesoramiento de la Dra. Fabiola Torres Adame y del Dr. Julio Pimienta Prieto. 


\section{Introducción}

Lograr una educación superior de calidad es un objetivo fundamental de cualquier sociedad. En este sentido se han llevado a cabo muchos esfuerzos por comprender y mitigar la deserción, por un lado, y por fomentar el aprendizaje y el buen desempeño académico de los estudiantes, por otro. A partir de los años ochenta se comenzó a fijar la atención también en un fenómeno que, según se ha demostrado, está estrechamente relacionado con los anteriores, el involucramiento estudiantil, entendido como el compromiso activo del estudiante con su propia experiencia formativa. En México, donde son recurrentes los estudios e informes que señalan datos alarmantes en términos de deserción y fracaso estudiantil, se han realizado también investigaciones que reflejan que existe poco involucramiento estudiantil (Torres, 2009).

Por otro lado, es creciente la atención que prestan las instituciones de educación superior a ofrecer una vida universitaria dinámica, repleta de opciones formativas como complemento al salón de clases. Se trata de las comúnmente denominadas actividades extracurriculares (deporte, arte y cultura, conferencias, acción social, entre otras). En muchos casos incluso este ámbito se convierte en el principal diferenciador ante la competencia, o cuando menos en una fuerte ventaja competitiva.

La presente investigación se planteó con el objetivo de profundizar en el fenómeno del involucramiento estudiantil desde un nuevo enfoque, siguiendo además las sugerencias de otros autores. Por un lado, centrándose en el involucramiento en lo que a actividades extracurriculares se refiere; por otro, al hacerlo esencialmente de forma cualitativa -hasta ahora han predominado los estudios cuantitativos- (De Garay, 2004; Torres, 2009); también por permitir la comparación entre dos extremos, alumnos involucrados y alumnos no involucrados (Ríos, 2004; Flores, 2005) y finalmente por incluir, además de alumnos, a otros actores importantes y generalmente excluidos de este tipo de estudios, como es el caso de profesores y administrativos (Flores, 2005).

Los hallazgos de la investigación demuestran que las actividades extracurriculares son un elemento muy importante en el ámbito universitario por cuanto contribuyen a la formación integral del joven y generan una atractiva vida universitaria. No obstante, dichas actividades deben quedar en un segundo plano en relación a la academia, como complemento de la misma. La existencia de una fuerte apatía estudiantil se revela como un fenómeno estrechamente relacionado en sentido negativo con el involucramiento extracurricular y que marca en gran medida la experiencia universitaria de los estudiantes.

Los factores que pueden determinar el involucramiento se engloban en tres grandes categorías: el propio estudiante (madurez, personalidad, intereses, etc.), el entorno del estudiante (familia, amistades, contexto educativo y cultural del país, etc.) y la institución (ambiente, oferta de actividades, recursos, fomento, etc.). En cuanto a las consecuencias, las más importantes son el desarrollo personal y la formación integral del joven así como un mayor sentido de pertenencia e integración con la comunidad universitaria. Por tipo de actividad extracurricular, las de carácter académico (conferencias, congresos...) son las mejor valoradas, mientras que las de pastoral o la participación en sociedades de alumnos y grupos de liderazgo son las que se consideran como menos importantes.

El estudio se realizó en una universidad privada católica de elite de la Ciudad de México y se trata de una tesis doctoral (ya presentada y aprobada) del programa 
"Liderazgo y dirección de instituciones de educación superior", de la Universidad Anáhuac México Norte.

\section{Marco teórico}

El constructo involucramiento estudiantil fue desarrollado por primera vez por Astin (1984), quien, estudiando el fenómeno de la deserción escolar, descubrió que el principal factor para evitar el abandono académico pasaba por la participación comprometida y activa del estudiante en el proceso formativo. Así, Astin define el involucramiento estudiantil como la cantidad de energía física y psicológica que el estudiante dedica a su experiencia universitaria. Partiendo de esta consideración, se puede decir que un estudiante involucrado, en términos generales, sería aquél que participa activamente en clase, que estudia o complementa su aprendizaje fuera de ella, que participa en actividades extracurriculares e interactúa frecuentemente con profesores y otros estudiantes.

Además de Astin, se consideró a otros autores para complementar y matizar la definición del involucramiento estudiantil, como es el caso de Russell, Ainley \& Frydenberg (2006), quienes subrayan la diferencia entre motivación (energía) e involucramiento (energía en acción); de Furlong \& Christenson (2008), que señalan que estamos ante un constructo multidimensional que comprende pensamientos, sentimientos y comportamientos del estudiante; de Coates (2006), quien pone el énfasis en la calidad del tiempo del estudiante (aprovechamiento real del tiempo dedicado a una actividad formativa), por encima de la cantidad, o Arguedas (2010), que habla de tres niveles de involucramiento (conductual, emocional y cognitivo).

Por el marco contextual en el que se llevó a cabo la investigación se prestó un especial interés a los estudios previos realizados en México. Al respecto, destaca la labor de Adrián De Garay (2001), quien hace una completa investigación sobre los hábitos sociales y culturales del universitario. También hay que mencionar a Flores (2005), quien se centró en los factores de involucramiento en estudiantes de alto desempeño académico, y, más recientemente, a Torres (2009), quien en su estudio para medir el nivel de involucramiento del universitario mexicano concluyó que éste se da en un grado muy bajo.

En cuanto al constructo actividad extracurricular, se toma la definición de Moriana et al. (2006), quienes lo definen como toda actividad desarrollada dentro del ámbito educativo como complemento para el alumno a la formación recibida en el salón de clases, y generalmente dependiente de la institución, planificada y desarrollada por ésta. Esta definición se complementa con la aportación de Brown (1999), quien hace hincapié en que toda actividad extracurricular debe satisfacer dos condiciones: no ser parte del programa curricular regular de la institución y tener cierta estructura y misión formativa que trascienda la mera socialización.

Sobre la relevancia de estas actividades en el ámbito universitario se manifiesta con mucha claridad Tinto (2003), al asegurar que el involucramiento en actividades extracurriculares debe ser la norma y no una excepción de la experiencia estudiantil. Hernández et al. (1999) y Pascarella y Terenzini (2005) dan mayor sustento a esa idea al llamar la atención sobre el hecho demostrado de que el impacto de las instituciones educativas en los estudiantes no se debe a una única experiencia (el currículo formal), sino a un cúmulo de experiencias interrelacionadas, tanto en el salón de clases como fuera del mismo. 


\section{Metodología}

El objetivo central del estudio es contribuir al conocimiento del fenómeno del involucramiento estudiantil del universitario, específicamente en lo que se refiere a su involucramiento en actividades extracurriculares. Para ello se pretende:

- Conocer las valoraciones de los estudiantes, así como de profesores y personal administrativo, en relación al fenómeno.

- Averiguar qué factores consideran los participantes que puede motivar el que un estudiante decida o no involucrarse en actividades extracurriculares.

- Detectar qué consecuencias trae consigo el involucramiento estudiantil en actividades extracurriculares.

- Identificar qué prioridad otorgan los participantes a los diversos tipos de actividades extracurriculares.

- Proponer acciones para que las instituciones de educación superior desarrollen una labor efectiva en relación a su oferta de actividades extracurriculares y fomento del involucramiento estudiantil en las mismas.

El tipo de estudio es esencialmente de corte cualitativo, aunque se realizó un pequeño cuestionario para su análisis cuantitativo.

El estudio se realizó fundamentalmente a través de sesiones de grupos focales (ver guías de tópicos en los anexos A) y B). El grupo focal se basa en la interacción discursiva y la contrastación de las opiniones de sus miembros, reflejando así la pluralidad de actitudes, experiencias y creencias que se pueden dar en un determinado grupo de personas en torno a un tópico y, por tanto, la visión conjunta del mismo (Martínez, 2006). De manera complementaria se aplicó el ya mencionado cuestionario.

Los participantes en el estudio fueron alumnos, profesores y personal administrativo. En total, participaron 75 personas (36 alumnos -18 involucrados y 18 no involucrados-, 14 profesores y 16 administrativos). Hubo equidad de género, realizándose cuatro grupos focales con mujeres y otros cuatro con hombres. En cuanto a las áreas académicas de adscripción se buscó igualmente representatividad, de forma que todas estuvieran incluidas en mayor o menor medida de acuerdo a su tamaño real (cantidad de alumnos y de profesores totales de cada escuela o facultad).

Los estudiantes son considerados como la fuente de información más importante del estudio. Se trabajó con ellos clasificados en dos grandes grupos: estudiantes muy involucrados en actividades extracurriculares y estudiantes poco o nada involucrados. Este planteamiento de considerar los opuestos es sugerido por algunos autores (Ríos, 2004; Flores, 2005), ya que al estar representados los dos extremos, se pueden comparar los resultados e identificar y analizar las diferencias, enriqueciendo con ello la investigación.

Por otro lado, se trabajó con dos grupos de profesores y con dos grupos de personal administrativo, pues se trata de personas que tienen una gran incidencia en la vida universitaria del estudiante. Ellos también son protagonistas del fenómeno y representan por así decirlo el otro lado de la moneda, pues por lo general son quienes organizan o promueven las actividades extracurriculares en las que se quiere que participe y se involucre el alumno. Por tanto, sus puntos de vista complementan la investigación de forma significativa. 
Por el enfoque cualitativo del estudio, que busca más representatividad que generalización, los participantes de la muestra fueron seleccionados de forma intencional, bajo los criterios que se consideraron convenientes para alcanzar los objetivos de la investigación. Así, por ejemplo, en el caso de los alumnos se consultó a coordinadores de carrera y a los responsables de las diversas áreas de vida estudiantil, quienes proporcionaron la información de qué alumnos suelen participar en actividades extracurriculares y cuáles no. Del mismo modo se trabajó con un grupo accesible, donde se buscó primar más la profundidad alcanzada en la información recolectada que el número de casos.

Una vez recopilada la información de los grupos focales y de las entrevistas individuales, ésta se organizó, procesó y analizó siguiendo la propuesta de Martínez (2006), que responde a cuatro etapas: categorización, estructuración, teorización y contrastación. Para ello se trabajó con apoyo del software informático Atlas.ti.

En lo que respecta al cuestionario se requirió otro tipo de análisis al tratarse, como ya se comentó, de un instrumento cuantitativo. En este caso, se presenta una distribución de frecuencias de acuerdo al número de menciones que cada actividad tuvo entre las tres primeras (1-3) y entre las tres últimas (8-10) y, con base en ello, se obtuvieron los porcentajes correspondientes a dichas menciones. Es decir, el porcentaje de participantes que valoró cada actividad en uno y otro extremo.

Esta información se presentó en cinco tablas, una por cada grupo de participantes (estudiantes involucrados, estudiantes no involucrados, profesores y administrativos) y otra más contemplando a todos los participantes (ver tabla 1). Así, se pudo ordenar las actividades según los porcentajes obtenidos y sacar las conclusiones pertinentes.

\section{Resultados y conclusiones}

\subsection{Respecto a conocer las valoraciones de estudiantes, profesores y perso- nal administrativo en relación al fenómeno}

Para los participantes en el estudio, la prioridad de una universidad debe ser la vida académica y, en segundo lugar, el tener una vida universitaria dinámica y atractiva (con diversas opciones para el tiempo libre, una oferta variada y de calidad de actividades extracurriculares y una buena convivencia e integración estudiantil). En tercer lugar mencionan a la formación profesional, enfocada a la incorporación del universitario al campo laboral.

Todos los grupos de participantes (alumnos muy involucrados y poco o nada involucrados en actividades extracurriculares, profesores y personal administrativo), coinciden en considerar a las actividades extracurriculares como un elemento muy importante en el contexto universitario por cuanto generan vida universitaria, convivencia entre la comunidad estudiantil, una sana distracción necesaria para los alumnos y una herramienta idónea para lograr la formación integral del joven. Asimismo, lo consideran algo propio a la esencia de las universidades.

No obstante, matizan que el papel de estas actividades no debe ser más importante que el de la formación dentro del salón de clase y del plan de estudios de cada carrera. Asimismo, subrayan la participación en estas actividades debe ser siempre por libre elección y en ningún caso, obligatoria. 
Los profesores son el grupo que otorga menor valor a las actividades extracurriculares, supeditándolas a su vinculación con lo académico (como complemento al aprendizaje o como reforzamiento de aptitudes necesarias para el ejercicio profesional).

\subsection{Respecto a averiguar qué factores consideran los participantes que pue- den motivar el que un estudiante decida o no involucrarse en actividades extracurriculares}

Se observan tres grandes categorías de factores: el propio estudiante, el entorno del estudiante y la institución. Hay una fuerte coincidencia con la revisión de la literatura realizada y en concreto se da una gran similitud con la clasificación de Torres (2009), que habla de tres grandes factores que determinan el grado de involucramiento del estudiante: aspectos institucionales, experiencias interpersonales y características del estudiante. En cuanto al papel de cada una, el propio estudiante es el factor más importante en términos generales y, de forma concreta, para estudiantes involucrados y para profesores. Sin embargo, para estudiantes no involucrados y para los administrativos responsables de planeación y ejecución de la oferta extracurricular, el factor dominante es la institución (en términos de interés y apoyo hacia el estudiante, en el caso de los primeros, y en términos de atractivo y buena organización de la oferta extracurricular, para los segundos). Por su parte, para los administrativos el factor más importante es el entorno del joven.

Lo anterior refleja que, mientras para los estudiantes involucrados ellos mismos son el principal factor de su involucramiento, es decir, es una cuestión interna, de convicción, que predomina sobre circunstancias externas; para los estudiantes no involucrados los principales factores son externos (la institución y el entorno), de forma que, si éstos no son favorables, se imposibilita el involucramiento. De esto se puede concluir que para los estudiantes involucrados el involucrarse es una meta y deseo personal, por lo que ante cualquier obstáculo se busca una solución o un modo de evitar que influya. Por su parte, para los estudiantes no involucrados, que parecieran no tener un fuerte interés personal por involucrarse en el ámbito extracurricular, cualquier impedimento es una buena excusa para no hacerlo.

Dentro de la categoría "el propio estudiante" cabe destacar el factor personalidad o carácter, fundamentalmente en cuanto a capacidad y gusto del joven por socializar, por relacionarse con otros compañeros.

Otros factores muy importantes dentro de esta categoría son: los gustos e intereses del estudiante, su madurez (entendida en sus aspiraciones en la vida y de ser consciente tanto de su responsabilidad como universitario como del valor del ámbito extracurricular) y el grado de integración y de sentido de pertenencia con la institución.

El entorno del estudiante como factor comprende a la familia del joven, su grupo de pares y amistades, la formación escolar previa y el contexto cultural y educativo nacional, de México.

El contexto cultural y educativo nacional es un factor del que no hay antecedentes en el marco teórico referencial y que hace referencia, en un sentido negativo, a la percepción de que en México no existe una tradición y formación de participar en actividades extracurriculares, como sí ocurre en otros países, fundamentalmente Estados Unidos. Al respecto no contribuyen ni las instituciones educativas, desde la época escolar, ni tampoco la idiosincrasia del mexicano, bajo el entendido de que en términos generales tiende a la apatía en lugar de a una actitud de dinamismo y compromiso. 
Otro factor que se puede considerar dentro del entorno y que emerge como importante e igualmente como novedoso en relación a la literatura de referencia es el contexto generacional del estudiante y, relacionado con ello, su ritmo y estilo de vida (uso de tecnologías, hábitos sociales y culturales). Al igual que en el caso del contexto cultural y educativo, éste se manifiesta también en un sentido negativo respecto al involucramiento extracurricular.

En cuanto a la institución como factor, comprende principalmente a la propia oferta de actividades extracurriculares (calidad, variedad, organización) y, en mucha menor medid, al ambiente institucional (convivencia, instalaciones), al interés y apoyo institucional (fomento, motivación, aportación de recursos) y, en última instancia, al interés o involucramiento docente y administrativo.

Para los administrativos responsables de la planeación y ejecución extracurricular, como ya se mencionó, la oferta es el factor más relevante de involucramiento, de forma que si ésta es atractiva, responde al interés del joven y está bien organizada, de forma natural se irá dando un involucramiento en dicha actividad.

Conviene señalar que el lugar de residencia del estudiante, mencionado en el texto pionero de Astin sobre involucramiento estudiantil como el factor más relevante del involucramiento extracurricular y, posteriormente, por autores como Kuh et al. (1994) o Hernández et al. (1999), no se manifiesta como tal (no se menciona en ningún momento) en los hallazgos de este estudio, lo cual puede deberse a que en México no opera un esquema de residencias estudiantiles como en Estados Unidos.

Considerando estos resultados, algunas recomendaciones concretas para las Instituciones de Educación son:

- A la hora de planificar y desarrollar la oferta de actividades, considerar no sólo los objetivos formativos de la misma, sino también las expectativas, gustos e intereses del joven, pues en la medida en que éstos se vean satisfechos será más probable lograr una participación amplia y motivada.

- Dar la mayor proyección posible de estas actividades (por ejemplo, mediante la participación en concursos o en torneos deportivos, la exposición de trabajos artísticos, la representación de obras de teatro o de espectáculos musicales en foros externos al campus, etc.). Para el universitario es importante saber que la actividad en la que participa puede llegar a trascender más allá del propio grupo de personas involucradas en ella, convirtiéndose en un reto e incluso en algo aspiracional.

- Implementar estrategias que favorezcan la integración y sentido de pertenencia de los profesores, ya que muchos de ellos, sobre todo los que no son de planta, se sienten poco vinculados a la institución más allá de la materia que imparten.

- Crear conciencia en el profesor de la importancia del ámbito extracurricular (hacerles ver los diversos beneficios que trae consigo, inclusive en cuanto a desempeño académico) e invitarlo a ser promotor activo del mismo.

- Promover en el personal administrativo un mayor conocimiento de la oferta extracurricular de la institución para que puedan ser portavoces de la misma en la medida de lo posible, gracias a la interacción constante con estudiantes (sobre todo en el caso de secretarias y coordinaciones de escuelas y facultades). 


\subsection{Respecto a detectar qué consecuencias trae consigo el involucramiento estudiantil en actividades extracurriculares}

La principal consecuencia del involucramiento extracurricular es el desarrollo personal y la formación integral del estudiante (adquisición mediante la puesta en práctica de valores como el trabajo en equipo, la organización, la disciplina, etc.). Esta relación se encuentra en la literatura abordada por autores como García (1991), Martínez (2000) o Estupiñán (2007), entre otros.

Otra consecuencia es la generación o fortalecimiento del sentido de pertenencia del estudiante hacia su institución, en gran medida motivado por una mayor convivencia e integración de la comunidad estudiantil, algo que ya encontramos en los textos de Mahoney, Cairns y Farwer (2003), Willms (2003) y De Garay (2004), por citar algunos ejemplos.

También una mayor proyección profesional, debido muy especialmente al establecimiento de relaciones personales a futuro con otros compañeros con gustos e intereses afines y que en algún momento pueden convertirse en compañeros de negocios o proveedores de oportunidades de trabajo. Cabe señalar que este hallazgo aparece sólo de forma ocasional en la literatura, a través de los textos de Flores (2005), Rivera (2007) y Hozelweiss (2009), y en mención al impacto positivo que tienen las actividades extracurriculares de cara a adquirir competencias profesionales (trabajo en equipo, liderazgo, resolución de problemas...), no se había encontrado esa importancia en cuanto a relaciones con otros compañeros, como es el caso de este estudio.

Una sana distracción y relajación de las responsabilidades académicas. Esto trae consigo a su vez que el alumno, cuando debe volver a concentrarse en sus estudios, lo haga con una mejor actitud, lo que puede repercutir en un mejor desempeño académico. Aquí se da cierta novedad respecto al marco teórico, ya que la relación involucramiento extracurricular-desempeño académico es indirecto, mientras para muchos autores (Pascarella y Terenzini, 1991; Van Matre, Valentine y Cooper, 2000; Vargas, 2002; Flores, 2005), la relación es directa y tiene que ver con aprendizaje y con la adquisición de aptitudes para el estudio.

Únicamente en el caso de las entrevistas al personal administrativo responsable de la planeación y ejecución de las actividades extracurriculares, se menciona una posible consecuencia negativa de este involucramiento, que es la inversión de tiempo, que trae consigo esfuerzo, compromiso y discriminación, por cuanto es tiempo que se deja de dedicar a otras actividades. No obstante, lo matizan bajo el argumento de que es algo que se soluciona con una buena organización.

En cuanto a deserción o permanencia, que es probablemente la consecuencia del involucramiento más recurrente en los referentes teóricos (Astin, 1984; Brown, 1999; Tinto, 2003; Flores, 2005; Jablon y Wilkinson, 2006; Huesca y Castaño, 2007; Wadley y Williford, 2008), no aparece en los resultados de nuestra investigación. Esto se puede deber al enfoque del estudio, donde no se trabajó con estudiantes desertores sino con estudiantes ya en buena medida consolidados (pues no se incluyó a alumnos de primer semestre) dentro de la UAMS. 


\subsection{Respecto a identificar qué prioridad otorgan los participantes a los diver- sos tipos de actividades extracurriculares ${ }^{2}$}

Como se puede apreciar en la tabla 1, para los participantes en el estudio, las actividades académicas complementarias (conferencias, congresos, concursos, proyectos, etc.) son con mucha diferencia respecto a las demás, el tipo de actividad extracurricular más importante. No obstante, considerando también a las tres actividades no extracurriculares incluidas en el cuestionario, participar activamente en clase se revela como la más importante. De esta forma se consolida el hallazgo según el cual a nivel universitario lo académico debe primar siempre sobre lo demás.

\begin{tabular}{|c|c|c|c|c|}
\hline Actividad & $\begin{array}{l}\mathrm{N}^{\circ} \text { de partici- } \\
\text { pantes } \\
\text { que consideran } \\
\text { a la actividad } \\
\text { entre las más } \\
\text { importantes } \\
\text { (extremo 1-3) }\end{array}$ & $\begin{array}{c}\mathrm{N}^{\circ} \text { de participan- } \\
\text { tes que conside- } \\
\text { ran a la actividad } \\
\text { dentro de las me- } \\
\text { nos importantes } \\
\text { (extremo 8-10) }\end{array}$ & $\begin{array}{l}\% \text { de participan- } \\
\text { tes que conside- } \\
\text { ran a la actividad } \\
\text { dentro de las } \\
\text { más importantes } \\
\text { (extremo 1-3) }\end{array}$ & $\begin{array}{l}\% \text { de participan- } \\
\text { tes que conside- } \\
\text { ran a la actividad } \\
\text { dentro de las me- } \\
\text { nos importantes } \\
\text { (extremo 8-10) }\end{array}$ \\
\hline $\begin{array}{l}\text { Participar activa- } \\
\text { mente en clase }\end{array}$ & 42 & 5 & $68,9 \%$ & $8,2 \%$ \\
\hline $\begin{array}{c}\text { Actividades } \\
\text { académicas } \\
\text { complementarias }\end{array}$ & 34 & 5 & $55,7 \%$ & $8,2 \%$ \\
\hline $\begin{array}{l}\text { Irse un semestre } \\
\text { de intercambio }\end{array}$ & 23 & 22 & $37,7 \%$ & $36,1 \%$ \\
\hline $\begin{array}{c}\text { Estudiar para un } \\
\text { examen }\end{array}$ & 17 & 19 & $27,9 \%$ & $31,1 \%$ \\
\hline $\begin{array}{l}\text { Actividades de } \\
\text { difusión cultural }\end{array}$ & 16 & 15 & $26,2 \%$ & $24,6 \%$ \\
\hline $\begin{array}{l}\text { Sociedad de } \\
\text { alumnos o gru- } \\
\text { pos de liderazgo }\end{array}$ & 16 & 24 & $26,2 \%$ & $39,3 \%$ \\
\hline $\begin{array}{l}\text { Programas de } \\
\text { acción social }\end{array}$ & 15 & 12 & $24,6 \%$ & $19,7 \%$ \\
\hline $\begin{array}{l}\text { Actividades } \\
\text { deportivas }\end{array}$ & 10 & 16 & $16,4 \%$ & $26,2 \%$ \\
\hline
\end{tabular}

2 Al término de los grupos focales se les entregó a los participantes un cuestionario en el que se les pedía priorizar entre diez actividades propias de la experiencia universitaria: siete son las de carácter extracurricular más comunes (deportes, difusión cultural, acción social, pastoral, actividades de carácter académico, intercambio, sociedades de alumnos y grupos de liderazgo) y tres que, sin ser extracurriculares, sí son propias de la etapa universitaria y determinan, junto al ámbito extracurricular, la diversidad de opciones que tiene un estudiante de ocupar su tiempo en el contexto universitario (participar activamente en clase, socializar con otros estudiantes en la cafetería y estudiar para un examen). Con ello se pretendió un doble objetivo: identificar la priorización que hacen los participantes de las actividades extracurriculares comparadas entre sí y al mismo tiempo comparadas con estas otras actividades tan comunes en la vida universitaria. De este modo se esperaba complementar además los hallazgos obtenidos del ejercicio planteado en los grupos de foco sobre la "universidad ideal", ya que las respuestas arrojarían información valiosa sobre los aspectos en los que los participantes centran el mayor interés al hablar del mundo universitario (lo académico, lo extracurricular, lo social). 


\begin{tabular}{|c|c|c|c|c|}
\hline Actividad & $\begin{array}{c}\mathbf{N}^{\circ} \text { de partici- } \\
\text { pantes } \\
\text { que consideran } \\
\text { a la actividad } \\
\text { entre las más } \\
\text { importantes } \\
\text { (extremo 1-3) }\end{array}$ & $\begin{array}{c}\mathbf{N}^{\circ} \text { de participan- } \\
\text { tes que conside- } \\
\text { ran a la actividad } \\
\text { dentro de las me- } \\
\text { nos importantes } \\
\text { (extremo 8-10) }\end{array}$ & $\begin{array}{c}\text { \% de participan- } \\
\text { tes que conside- } \\
\text { ran a la actividad } \\
\text { dentro de las } \\
\text { más importantes } \\
\text { (extremo 1-3) }\end{array}$ & $\begin{array}{c}\% \text { de participan- } \\
\text { tes que conside- } \\
\text { ran a la actividad } \\
\text { dentro de las me- } \\
\text { nos importantes } \\
\text { (extremo 8-10) }\end{array}$ \\
\hline $\begin{array}{c}\text { Socializar con } \\
\text { otros estu- } \\
\text { diantes en la } \\
\text { cafetería }\end{array}$ & 5 & 34 & $8,2 \%$ & $\mathbf{5 5 , 7 \%}$ \\
\hline Pastoral & 4 & 31 & $6,6 \%$ & $\mathbf{5 0 , 8 \%}$ \\
\hline
\end{tabular}

Tabla 1. Distribución de frecuencias del cuestionario de priorización de actividades propias a la experiencia universitaria, por nivel de importancia. Concentrado de estudiantes, profesores y administrativos. Fuente: Elaboración propia en base al cuestionario aplicado en la investigación.

Cabe destacar que irse un semestre de intercambio es la segunda actividad extracurricular con mayor porcentaje de valoración positiva, aunque con el matiz de que, al mismo tiempo, obtiene también un porcentaje casi idéntico en el extremo negativo, lo que puede explicarse por tres motivos. Por un lado a que pudiera haber estado bien valorado por los participantes que vivieron esa experiencia o tuvieron esa intención, y mal valorado por quienes nunca lo vivieron o contemplaron. Relacionado con esto, puede deberse también, en sentido negativo, a que se valore esta actividad como elitista, destinada a pocos estudiantes y, por tanto, de impacto limitado. Finalmente, puede deberse a la percepción sobre el aprovechamiento de la actividad, ya que puede haber quien lo considere como una oportunidad única de aprendizaje y desarrollo o quien lo considere como una oportunidad para la fiesta y diversión que aporte poco o nada en la formación del universitario.

Por el contrario, las actividades extracurriculares consideradas como menos relevantes, o menos valoradas en relación a las demás, son las de pastoral, bajo el argumento de que se trata de un ámbito demasiado personal con lo que no necesariamente todos los alumnos se identifican, y que por tanto no corresponde al contexto universitario sino al contexto de la vida familiar y social de cada estudiante. Este hallazgo es relevante por cuanto, en el caso de la UAMS, el desarrollo de una pastoral universitaria protagonista de la vida estudiantil es un objetivo de primer nivel.

La segunda actividad extracurricular con una baja valoración es el formar parte de una sociedad de alumnos o grupo de liderazgo. Esto puede deberse a que ninguno de los estudiantes no involucrados había formado parte de alguno de estos grupos; y también al hecho de que, desde el punto de vista de esos estudiantes así como de profesores y administrativos, pueden verse como grupos elitistas que no impactan en la mayoría del alumnado.

Incluyendo en el análisis a las tres actividades no extracurriculares, socializar con otros estudiantes en la cafetería emerge como la peor valorada, lo que puede deberse a que en términos generales, para los participantes, lo más importante en el paso por la universidad son aquellas actividades que pueden tener un impacto intencional en el estudiante, ya sea de carácter académico o de desarrollo personal, por encima de actividades como esta socialización, cuyo impacto no es intencional o no se encuentra estructurado. 


\section{Investigaciones futuras}

El enfoque y los resultados de esta investigación invitan a plantearse futuras investigaciones que la complementen y a través de las cuales se logre una mayor profundización en el conocimiento y comprensión del fenómeno. Por ejemplo:

1. Sería oportuno hacer nuevos estudios cualitativos en instituciones de diferentes características para poder comparar resultados, o incluso dentro de una misma universidad, hacer la diferenciación entre escuelas.

2. Realizar un estudio muy similar, también con un enfoque cualitativo y considerando los mismos objetivos de investigación, pero para profundizar en el otro lado de la moneda, el involucramiento estudiantil con la formación curricular, es decir, con el desarrollo normal del plan de estudios de su carrera universitaria (involucramiento en el salón de clases, con el repaso o estudio de los temas fuera del salón, con la preparación de exámenes, etc.).

3. Hacer también el mismo estudio cualitativo pero aplicado sólo a un tipo de actividad extracurricular (por ejemplo deportivas). Esto permitiría un nivel aún mayor de profundización, sumamente valioso para las instituciones a la hora de concretar una determinada oferta extracurricular.

4. Uno de los términos más mencionados en los grupos focales es el de apatía estudiantil. Por ello, sería relevante profundizar en este fenómeno mediante un estudio sociológico. ¿Qué entienden los universitarios exactamente por apatía?, ¿por qué están en la universidad?, ¿qué expectativas tienen?, etc.

5. Finalmente, sería interesante replicar este estudio en otros países y en instituciones de similares características con el fin de establecer comparaciones. ¿Tendrán una valoración similar del fenómeno?, ¿habrá factores "exclusivos" del contexto mexicano?, ¿cuál será la priorización de actividades extracurriculares en otros entornos?

\section{Referencias}

Arguedas, I. (2010). Involucramiento de las estudiantes y los estudiantes en el proceso educativo. Revista Electrónica Iberoamericana sobre Calidad, Eficacia y Cambio en Educación (REICE), 8 (1). Recuperado de http://www.rinace.net/reice/numeros/arts/ vol8num1/REICE\%20Vol8,1.pdf.

Astin, A. (1984). Student Involvement: A Developmental Theory for Higher Education. Graduate School of Education, University of California. Recuperado en septiembre de 2009 desde

http://www.middlesex.mass.edu/TutoringServices/Astinlnvolvement.pdf

Brown, R. (1999). Extracurricular activity: how does participation encourage positive youth development? University of Nevada, Reno. Recuperado de http://www.unce.unr.edu/ publications/files/cy/other/fs9932.pdf.

Coates, H. (2006). Student engagement: the specification and measurement of student engagement. En Student engagement: measuring and enhancing engagement with learning. Proceedings of a symposium. AAU Series on Quality, $n^{\circ} 10$, Nueva Zelanda. Recuperado de http://www.nzuaau.ac.nz/node/64. 
De Garay, A. (2001). Los actores desconocidos: una aproximación al conocimiento de los estudiantes. México: ANUIES.

Flores, J. (2005). El fascinante mundo de los estudiantes exitosos. Percepciones de estudiantes de licenciatura de alto desempeño, sobre los factores que influyen en su nivel de compromiso e involucramiento estudiantil. Universidad de Monterrey.

Furlong, M. y Christenson, S. (2008). Engaging students at school and with learning: a relevant construct for all students. Psychology in the schools, 45 (5).

Hernández, K., Hogan, S., Hathaway, C. y Lovell, C. (1999). Analysis of the literature on the impact of student involvement on student development and learning: more questions than answers? NASPA Journal, 36 (3).

Martínez, M. (2006). Ciencia y arte en la metodología cualitativa. México: Trillas.

Moriana, J., Alós, F., Alcalá, R., Pino, M., Herruzo, J. y Ruiz, R. (2006). Actividades extraescolares y rendimiento académico en alumnos de educación secundaria. Electronic Journal of research in educational psychology, 4 (8). Recuperado de http://dialnet. unirioja.es/servlet/articulo?codigo $=1983608$.

Pascarella, E. y Terenzini P. (2005). How college affects students: a third decade of research. San Francisco: Jossey-Bass.

Ríos, R. (2004). Universitarios y Voluntariado: Análisis del Involucramiento en Acciones Filantrópicas de los Alumnos de la PUC. Psykhe, 13 (2). Recuperado de http://redalyc. uaemex.mx/pdf/967/96713208.pdf.

Russell, J., Ainley, M. y Frydenberg, E. (2006). Schooling issues digest: student motivation and engagement. Recuperado de http://www.dest.gov.au/sectors/school_education/ publications_resources/schooling_issues_digest/schooling_issues_digest_motivation_engagement.htm.

Tinto, V. (2003). Student success and the building of involving educational communities. Syracuse University, Higher Education Monograph Series 1-11. Recuperado de http:// www.marin.edu/WORD-PPT/PromotingStudentSuccess.pdf.

Torres F. (2009). Involucramiento estudiantil del Universitario Mexicano y su Relación con el Desarrollo Académico y Personal. Tesis Doctoral, Universidad Anáhuac México Norte, México. 


\section{Pregunta, ejercicio, dinámica.}

Imagina que te invitan a planificar una universidad desde cero como parte del consejo fundador, ¿qué aspectos sugerirías como imprescindibles?, ¿por qué?

Cada vez es más común escuchar decir que "los estudiantes son apáticos, y lo único que les importa es pasar las materias como sea para obtener el título", ¿qué piensas de esto?

¿Qué pensarías si se decide que a partir del próximo semestre no habrá actividades extracurriculares en la universidad?

Piensen en un alumno o alumna que suele destacar por que le va muy bien en lo académico pero que además suele participar en otro tipo de actividades, como asistir a conferencias, practicar algún deporte, colaborar en acción social, etc. En fin, un alumno que suele estar muy metido en la vida universitaria, ¿cuál es tu opinión de ese perfil de alumno?, ¿por qué?

¿Y qué piensan del perfil contrario, de los alumnos que deciden sólo ir a clases y estudiar?, ¿por qué?

En ambos casos, ¿qué aspectos positivos o negativos creen que obtienen de ello?

¿A qué crees que se deba que algunos estudiantes, como el del caso anterior, participen en muchas actividades universitarias y otros no?

Y en particular, ¿qué hace que tú participes o no en una actividad extracurricular?, ¿cuáles son tus motivaciones?

En esta universidad, ¿creen que existe una buena disposición de los estudiantes para participar?, ¿por qué?

Si pidieran tu colaboración para elaborar la oferta de actividades extracurriculares del próximo semestre, ¿qué sugerirías y por qué?

¿Qué sueles hacer en la universidad cuando no estás en clase?

¿Y cuándo no estás en la universidad, a qué actividades sueles dedicar más tiempo?

Recuerda alguna actividad extracurricular que hayas realizado en la universidad y que te haya marcado especialmente, ¿qué actividad fue?, ¿por qué te marcó? Cuéntanos sobre la experiencia.

Anexo A. Guía de tópicos para los grupos focales con estudiantes.

Fuente: Elaboración propia con base en los objetivos de la investigación.

Guía validada por expertos. 


\section{Pregunta, ejercicio, dinámica.}

En tu experiencia como profesor o como administrativo, recuerda algún momento o alguna vivencia en la que hayas dicho "mi trabajo realmente vale la pena". En el que te hayas sentido especialmente satisfecho.

En el sentido opuesto, recuerda algún momento o vivencia en el que hayas dicho, “¿qué hago yo aquí?, ¿por qué me dedico a esto?”. En el que te hayas sentido especialmente molesto, frustrado o desilusionado.

Imagina que te invitan a planificar una universidad desde cero, la universidad ideal, como parte del consejo fundador, ¿qué aspectos sugerirías como imprescindibles?, ¿por qué?

Muchos profesores dicen que "los estudiantes son apáticos, y lo único que les importa es pasar las materias como sea para obtener el título", ¿qué piensan de esto?

Por favor completen esta frase tan extensamente como crean necesario: Los profesores (o administrativos) son importantes en la experiencia universitaria del alumno porque...

En general, ¿creen que la mayoría de profesores / administrativos se interesan por el estudiante y se comprometen con su formación?, ¿por qué?

Y, en el sentido inverso, ¿creen que los estudiantes aprovechan al máximo a sus profesores (o personal administrativo)?, ¿por qué?

Piensen en un alumno o alumna que suele destacar por que le va muy bien en lo académico pero que además suele participar en otro tipo de actividades, como asistir a conferencias, practicar algún deporte, colaborar en acción social, etc. En fin, un alumno que suele estar muy metido en la vida universitaria, ¿cuál es su opinión de ese perfil de alumno?, ¿por qué?

¿Y qué piensan del perfil contrario, de los alumnos que deciden sólo ir a clases y estudiar?, ¿por qué?

En ambos casos, ¿qué aspectos positivos o negativos creen que se deriva de ello?

¿A qué creen que se deba el que algunos estudiantes, como el del caso anterior, participen en muchas actividades universitarias y otros no?

¿Creen que ustedes los profesores / administrativos pueden influir para que los alumnos participen o no en actividades extracurriculares?

En esta universidad, ¿creen que existe una buena disposición de los estudiantes para participar e involucrarse?, ¿por qué?

Anexo B. Guía de tópicos para los grupos focales con profesores y administrativos.

Fuente: Elaboración propia con base en los objetivos de la investigación.

Guía validada por expertos. 